Journal of Molecular Structure (Theochem), 109 (1984) 351-366

Elsevier Science Publishers B.V., Amsterdam - Printed in The Netherlands

\title{
CALCULATIONS ON VIBRATIONAL PROPERTIES OF H-BONDED OH GROUPS, AS A FUNCTION OF H-BOND GEOMETRY
}

\author{
J. G. C. M. VAN DUIJNEVELDT-VAN DE RIJDT and F. B. VAN DUIJNEVELDT
}

Theoretical Chemistry Group, State University of Utrecht, P.O. Box 80.062; 3508 TB Utrecht (The Netherlands)

\section{J. A. KANTERS}

Structural Chemistry Group, State University of Utrecht, P.O. Box 80.062, 3508 TB Utrecht (The Netherlands)

D. R. WILLIAMS

University of Colurado, 1100 Fourteenth Street, Denver, CO 80202 (U.S.A.)

(Received 18 February 1984)

\begin{abstract}
Ab initio calculations on $\left(\mathrm{H}_{2} \mathrm{O}\right)_{2}$ have been performed on 10 model geometries and 17 geometries which simulate $\mathrm{O}-\mathrm{H}$...O contacts encountered in the crystals of $\beta$-D-fructose, $\beta$-D-arabinose and turanose. The variations with geometry of the hydrogen-bond energy $\Delta E$, the lengthening of the donor $\mathrm{OH}$ distance $(\Delta r)$, the frequency shift $\Delta \nu_{\mathrm{OH}}$ and the IR absorption intensity $I$ are investigated. The relation between $\Delta \nu_{\mathrm{OH}}$ and $\Delta r$ is discussed and it is found that most of the observed $\Delta \nu_{\mathrm{OH}}$ arises from $\Delta r$. It is shown that there is no one-to-one correspondence between $\Delta \nu_{\mathrm{OH}}, \Delta E$ and $I$. In particular, $\Delta E$ may be large even for cases where $\Delta \nu_{\mathrm{OH}}$ is small. The results are used to determine which $\mathrm{O}-\mathrm{H} \cdots \mathrm{O}$ contacts give rise to the narrow $\mathrm{OH}$ bands observed in the saccharide crystals.
\end{abstract}

\section{INTRODUCTION}

A hydrogen bond with H-bond energy $\Delta E$ can be associated with a variety of properties such as a short, nearly linear $\mathrm{O}-\mathrm{H}$. . O contact, an IR frequency shift $\Delta \nu_{\mathrm{OH}}$, a change in the IR absorption intensity $I$, a lengthening $\Delta r$ of the donor $\mathrm{OH}$ distance or a change in the electric field gradient at the $\mathrm{H}$ position.

Conversely, from the absence of a given property (usually $\Delta \nu_{\mathrm{OH}}$ ), it is frequently concluded that there is no $H$ bond. A well-known example is the IR and Raman absorption near $3600 \mathrm{~cm}^{-1}$ in liquid water, which is usually assigned to free $\mathrm{OH}$ groups [1]. Likewise, the narrow IR bands near $3600 \mathrm{~cm}^{-1}$ observed in certain crystals of saccharides and hydroxy acids have been advanced as a means for detecting non-bonded hydroxyl groups [2]. The validity of such interpretations depends on the assumption that there is a one-to-one correspondence between all the characteristic H-bond properties, so that any one of them can serve as the criterion for 
the presence or absence of a $\mathrm{H}$ bond. The object of the present paper is to show that such a one-to-one correspondence does not in general exist if one compares different geometries of a given $\mathrm{H}$-bonded system. In particular, we investigate how $\Delta \nu$ varies with geometry and we ask whether a small $\Delta \nu$ necessarily coincides with small values of $\Delta E$ and $I$.

This is done by calculating $\Delta E, I, \Delta \nu$ and $\Delta r$ for the $\mathrm{H}_{2} \mathrm{O}$ dimer using model geometries and geometries which simulate $\mathrm{O}-\mathrm{H} \ldots \mathrm{O}$ contacts in the crystals of mono- and disaccharides. The model is described in the next section, and the results in a subsequent section. In agreement with a suggestion of Lindgren and Tegenfeldt [3] we find that there is an almost linear relationship between $\Delta \nu$ and $\Delta r$, which is discussed later. Finally, we return to the narrow $\mathrm{OH}$ bands observed in the saccharide crystals and we ask which of the (many) $\mathrm{O}-\mathrm{H} \ldots \mathrm{O}$ contacts give rise to these sharp peaks, since such an assignment on the basis of simple geometrical criteria has proved to be impossible.

\section{DETAILS OF CALCULATIONS}

The calculations were performed on the 17 crystallographically unique $\mathrm{O}-\mathrm{H}$... O contacts occurring in the crystals of turanose, a disaccharide, [4] and the monosaccharides $\beta$-D-fructose [5] and $\beta$-D-arabinose [6]. All $17 \mathrm{OH}$ groups observed in these crystals were considered and within a radius of $3 \AA$ there was only one nearest-neighbour acceptor $O$ atom for each $\mathrm{OH}$ group. Five of these H-bond interactions were observed in $\beta$-D-fructose, which we call F1 to F5, eight in turanose (T1-T8) and four in $\beta$-D-arabinose (A1-A4). Each observed $\mathrm{R}_{1}-\mathrm{O}_{1}-\mathrm{H} \cdots \mathrm{O}_{2}-\mathrm{R}_{2}$ contact was modelled by a water-water contact, i.e., the observed bonds $R_{1}-O_{1}, R_{2}-O_{2}$ and $O_{2}-R_{3}$ were replaced by $\mathrm{OH}$ bonds of standard $\mathrm{OH}$ length (1.80887 a.u.). The angles and the $\mathrm{O}_{1} \ldots \mathrm{O}_{2}$ distance were kept fixed at the experimentally observed values, which accounts to some extent for chemical differences between the various $\mathrm{O}-\mathrm{H}$ donors and acceptors. These $17 \mathrm{O}-\mathrm{H} \ldots \mathrm{O}$ contacts form a small sample of all the $\mathrm{H}$-bond geometries which can be encountered experimentally. This sample covers a range of $\mathrm{H}$-bond distances and orientations but it is not large enough to isolate the influence of one particular geometrical parameter. We therefore also include results for calculations on the water dimer in a number (ten) of model geometries with $C_{\mathrm{s}}$ symmetry, to which we refer as $M 1-M 10$, which are designed specifically to bring out the dependence of various $\mathrm{H}$-bond properties on the geometrical parameters $R, \theta$ and $\chi$ (Fig. 1). These parameters show the influence of the acceptor distance, the acceptor orientation and the H-bond linearity, respectively. Our choice of these parameters was based on the observation that at a given $R$ the maximum $\Delta E$ can always be attained by imposing $C_{s}$ symmetry and varying $\theta$ and $x[7]$. 


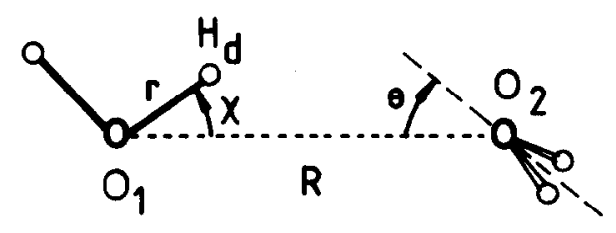

Fig. 1. Definition of the parameters $\chi, \theta$ and $R$ characterizing a $\left(\mathrm{H}_{2} \mathrm{O}\right)_{2}$ geometry.

In order to determine the $\mathrm{O}-\mathrm{H}$ vibrational properties of the $\mathrm{H}$-donor in a given $\mathrm{O}-\mathrm{H}$... $\mathrm{O}$ contact, the ab initio calculations were performed at four different $\mathrm{O}_{1}-\mathrm{H}_{\mathrm{d}}$ lengths, viz. $1.75887(0.05) \ldots 1.90887$ a.u. That is, the position of $\mathrm{O}_{1}$ was kept fixed while the position of $\mathrm{H}_{\mathrm{d}}$ was varied, since in the $\mathrm{O}-\mathrm{H}$ normal mode it is the $\mathrm{H}$ atom which is displaced most. For each $\mathrm{O}-\mathrm{H}$ length, the total energy $E_{\mathrm{d}}$ of the dimer was obtained as a sum of the SCF energy of the dimer, corrected for BSSE effects, and the dispersion energy [8]. The SCF energies $\left(E_{\text {don }}\right.$ and $\left.E_{\text {acc }}\right)$ for the separate $\mathrm{H}$ donor and acceptor molecules, each in its own basis set (these are different for each $\mathrm{OH}$... $\mathrm{O}$ contact) were also obtained. The donor $\mathrm{OH}$ equilibrium lengths for the monomer $\left(r_{\mathrm{m}}\right)$ and the dimer $\left(r_{\mathrm{d}}\right)$ and the corresponding donor $\mathrm{OH}$ force constants $k_{\mathrm{m}} \equiv\left(\partial^{2} E_{\mathrm{don}} / \partial r^{2}\right)_{r_{\mathrm{m}}}$ and $k_{\mathrm{d}} \equiv\left(\partial^{2} E_{\mathrm{d}} / \partial r^{2}\right)_{r_{\mathrm{d}}}$ were obtained from four-point interpolation on the four donor and dimer energies, respectively [9]. This gives the change in optimum OH length $\left(\Delta r \equiv r_{d}-r_{m}\right)$ and the change in force constant $\left(\Delta k \equiv k_{\mathrm{d}}-k_{\mathrm{m}}\right)$ on dimerization. Moreover, for further analysis, the dimer $\mathrm{OH}$ force constant was also calculated at the monomer $\mathrm{OH}$ length: $k_{\mathrm{d}}\left(r_{\mathrm{m}}\right)$. For comparison with experiment we note that the change in $\nu_{\mathrm{OH}}$ corresponding to a given $\Delta k$ may be estimated from $\Delta \nu_{\mathrm{OH}}\left(\mathrm{cm}^{-1}\right)=3300 \Delta k$ (a.u. $)$.

In addition to total dimer energies, interaction energies $\Delta E$ were obtained by subtracting the monomer energies $E_{\text {don }}$ and $E_{\text {acc }}$ from the total dimer energy $E_{\mathrm{d}}$. As in previous work [8] the interaction energy was further partitioned into a first-order contribution $\left(E^{1}\right)$, a second-order contribution $\left(E^{2}\right)$ and a dispersion term $\left(E^{\text {disp }}\right)$. Here $E^{1}$ is the sum of the Coulomb $\left(E^{\mathrm{Coul}}\right)$ and the exchange energy $\left(E^{\text {exch }}\right)$ whereas $E^{2}$ consists of an induction $\left(E^{\text {ind }}\right)$ and a charge transfer $\left(E^{\mathrm{CTX}}\right)$ part. Finally, for each dimer we determined the $\mathrm{O}-\mathrm{H}$ dipole derivatives, and from these we calculated the expectation value of $(\partial \vec{\mu} / \partial r \cdot \partial \vec{\mu} / \partial r)$ where $\mu$ is the dipole moment of the dimer and $r$ the $\mathrm{OH}$ length. These numbers will be labelled $I$ since they are approximately proportional to the intensities of the $\mathrm{O}-\mathrm{H} v \rightarrow(v+1)$ IR transitions. In strong H-bonds $I$ is about ten times larger than in a monomer and so $I$ and $\Delta I$, the increase upon dimerization, are numerically similar. In the following we have chosen to tabulate $I$ rather than $\Delta I$.

The various monomer and dimer calculations were performed with local versions of IBMOLH [10]. The Coulomb, induction and dispersion energies were calculated with the program INTACT [11]. The atomic basis set employed was the split-valence SV basis, described in ref. 12. Preliminary 
calculations showed that the results obtained by this basis set for various properties $(\Delta r, \Delta k$ and $I)$ are not as accurate as those obtained by the larger polarized DZP and DZPP bases [12]. However, the SV basis meets our requirements, since our aim is to study qualitative correlations. Moreover, since we consider dimers rather than the full crystalline environments of each $\mathrm{OH}$ group, even the use of a larger basis set would not yield quantitative results for the properties of interest. For completeness we note that the SV basis correctly reproduces the large $I$ values of strong $\mathrm{H}$ bonds (e.g., A1), but for weak $\mathrm{H}$ bonds and monomers $I$ is too low. These findings parallel the results obtained by Zilles and Person in ab initio calculations of $\mathrm{OH}$ frequencies using the 4-31G basis set [13].

\section{VARIATION OF H-BOND PROPERTIES WITH H-BOND GEOMETRY}

In this section we discuss how the H-bond properties such as $\Delta E$ and $\Delta k$ vary as the $\mathrm{H}_{2} \mathrm{O}$ dimer geometry is changed. We first consider the model geometries M1-M10 which bring out the role of $R, \theta$ and $\chi$ (Fig. 1). Figure 2 shows the changes in $\Delta E, \Delta k$ and $I$ if one varies the acceptor angle $\theta$ for a linear, near-equilibrium H-bond geometry $\left(\chi=0^{\circ}, R=2.9 \AA\right)$. It is seen that the $\Delta k$ and $I$ curves are very nearly symmetrical around $\theta=0^{\circ}$ while $\Delta E$ shows the well-known optimum near $\theta=+50^{\circ}$. This behaviour is to be expected, since the forces on the donor proton arising from the acceptor molecule are much the same whether $\theta$ is positive or negative and hence the $\Delta k$ curve will be symmetrical around $\theta=0^{\circ}$. The same argument applies for the forces due to the acceptor on the electron distribution around the donor proton, which are responsible for the change in the dipole derivative $\partial \mu / \partial r$ and so $I$ is symmetrical around $\theta=0^{\circ}$. The $\Delta E$ curve, however, is not symmetrical about $\theta=0^{\circ}$, since for negative $\theta$ the acceptor $\mathrm{H}$ atoms lie on the

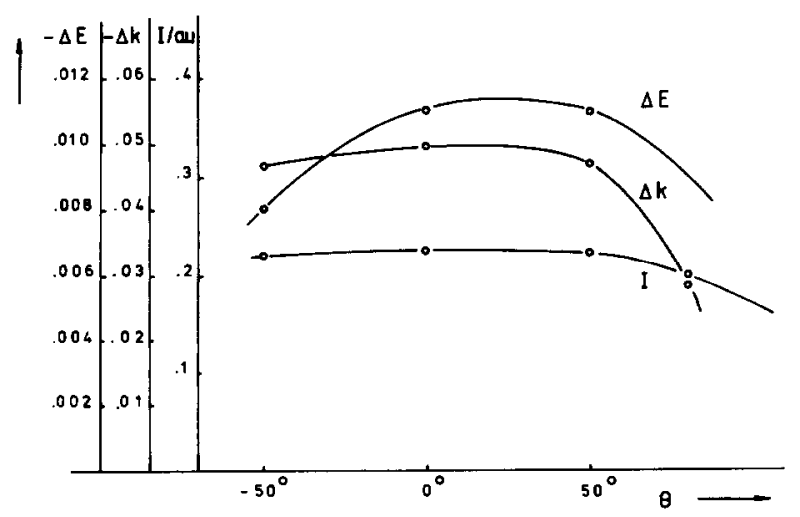

Fig. 2. Dependence of $\Delta E, \Delta k$ and $I$ (all in a.u.) upon the acceptor orientation for the model $\left(\mathrm{H}_{2} \mathrm{O}\right)_{2}$ dimers. 
same side of the $\mathrm{O} \cdots \mathrm{O}$ axis as the donor off-axis $\mathrm{H}$ atom, and are repelled by it.

In Fig. 3 the effect of $H$-bond non-linearity is studied by varying $\chi$ for near-equilibrium values of $R$ and $\theta\left(\theta=+50^{\circ}, R=2.9 \AA\right) . \Delta E, I$ and $\Delta k$ are all much more sensitive to a $10^{\circ}$ change in $\chi$ than to a $10^{\circ}$ change in $\theta$. The $\Delta E$ curve is roughly symmetrical about $\chi=0^{\circ}$ as a result of a compromise between the Coulomb energy, which is lowered for negative $\chi$, and the remaining energy terms, which favour positive $\chi$.

$I$ is also roughly symmetrical about $\chi=0^{\circ}$, rapidly decreasing for $|\chi|>10^{\circ}$. Its behaviour closely resembles that of $E^{2}$ and $E^{\text {exch }}$ (cf. Table 1). The $\Delta k$ curve, however, is strikingly unsymmetrical. For $\chi$ in the range $0-20^{\circ}, \Delta k$ is large, but for $\chi=-20^{\circ} \Delta k$ is almost reduced to zero, i.e., no shift in $\mathrm{OH}$ frequency occurs, even though the H-bond energy is only 0.003 a.u. smaller than for $\chi=0^{\circ}$.

Finally, Fig. 4 shows the influence of varying the intermolecular distance $R$ for a linear $\mathrm{H}$ bond $\left(\chi=0^{\circ}\right.$ and $\left.\theta=50^{\circ}\right) . \Delta E$ and $\Delta k$ show parallel behaviour, reaching a maximum value near $2.9 \AA$ and $2.8 \AA$, respectively. For $\Delta E$ this is the expected behaviour, corresponding to dominating exchange repulsion $\left(E^{\mathrm{exh}}\right)$ at short $R$. Analysis of the forces contributing to $\Delta k$ (cf. the following section) show that here, too, short-range repulsion dominates at short $R$ and prevents a further decrease of the force constant $k$. This result shows that the well-known inverse relation between the average $\nu_{\mathrm{OH}}$ and $R$ values [14] for a series of different H-bonding systems (ethers, alcohols, etc.) does not hold when considering different $R$ values of a given system (in our case, $\mathrm{H}_{2} \mathrm{O}$ dimer). Rather, $\Delta k$ (and hence $\nu_{\mathrm{OH}}$ ) is found to be a flat function of $R$ in the region near the equilibrium geometry $(R=2.9 \AA)$.

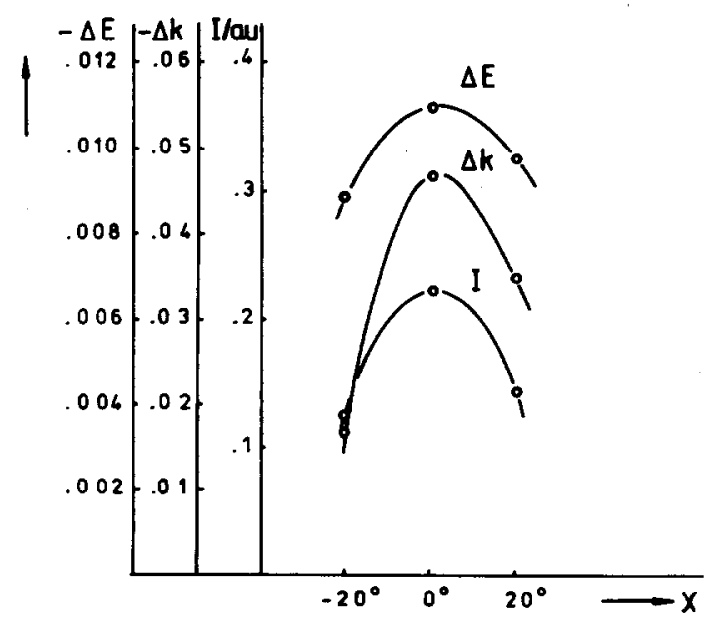

Fig. 3. Dependence of $\Delta E, \Delta k$ and $I$ upon the non-linearity $\times$ for the model $\left(\mathrm{H}_{2} \mathrm{O}\right)_{2}$ dimers. 


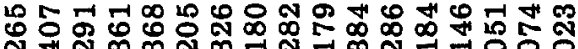
స స ल ల

$-$

童

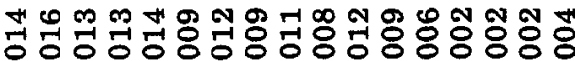

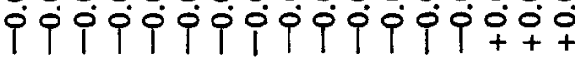

以

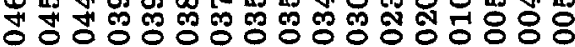

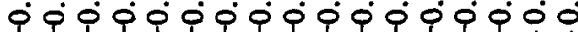

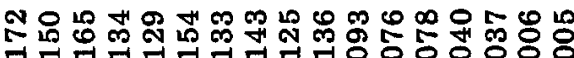

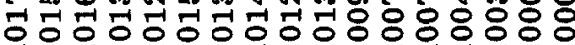
000000000000
+++++++1000

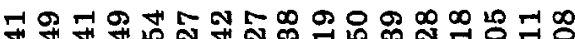

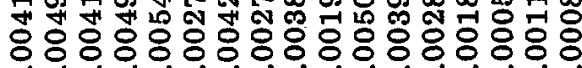

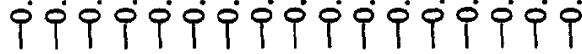

$0 \infty 0$ 足 น

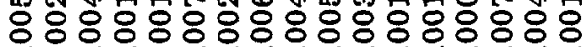

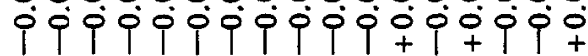

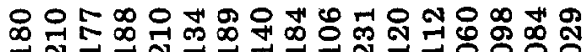

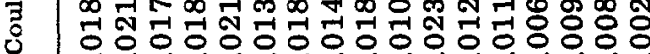
国

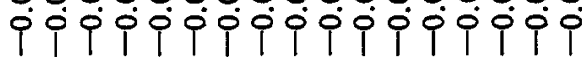

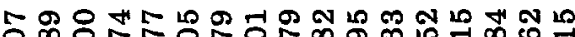

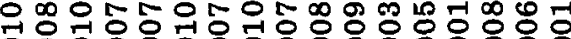

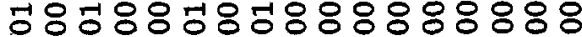

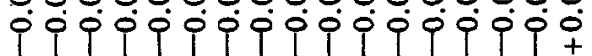

ᄂ

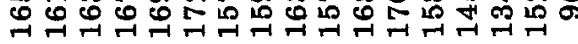

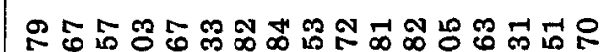

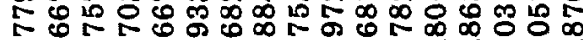

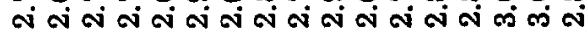

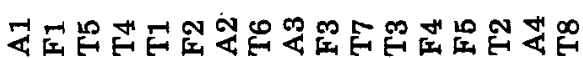

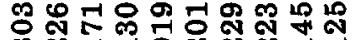

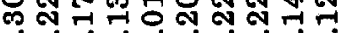

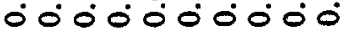

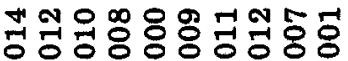

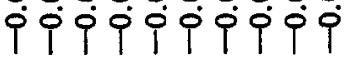

둔

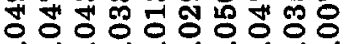

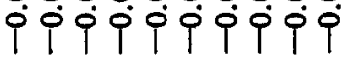

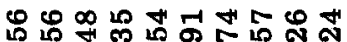

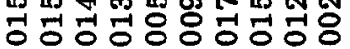
○ं $\dot{0} 0 \dot{0} \dot{0} \dot{0} 0 \dot{0} 0 \dot{0}$ t t + t t + t t t

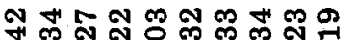

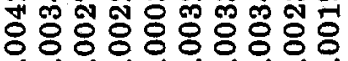

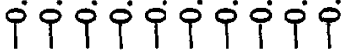

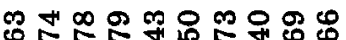

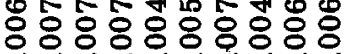

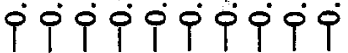

Нด न1 i i i i

ฟ

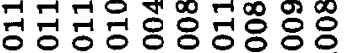

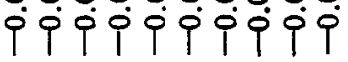

-00000000

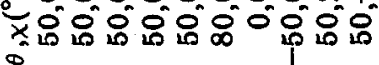

co 0 \% ง ต ल

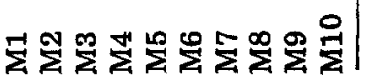




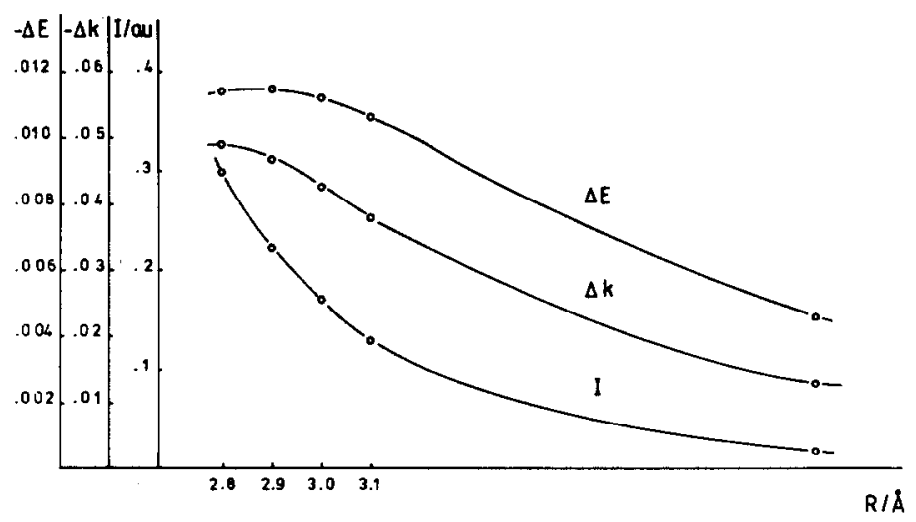

Fig. 4. Dependence of $\Delta E, \Delta k$ and $I$ upon $R$ for the model $\left(\mathrm{H}_{2} \mathrm{O}\right)_{2}$ dimers.

One of the consequences of this result is that the usual explanation for the breadth of the $\nu_{\mathrm{OH}}$ band in, for example, liquid water, in terms of a distribution of $R$ values [15] must be questioned. On the basis of the results presented above we suggest that a more likely cause for the breadth will be the distribution of angles such as $\theta$ and, especially, the non-linearity $\chi$.

Figure 4 also shows that the IR intensity $I$ increases exponentially with decreasing $R$. As for the $\chi$-dependence, this behaviour is parallel to the increase of $E^{2}$ and $E^{\text {exch }}$ (cf. Table 1). These observations suggest that for a collection of $\left(\mathrm{H}_{2} \mathrm{O}\right)_{2}$ geometries $I$ may be proportional to $E^{2}$ and/or $E^{\text {exch }}$. We return to this point below.

Let us now discuss the $\Delta E, I$ and $\Delta k$ values of the 17 experimentally observed $\mathrm{O}-\mathrm{H} \ldots \mathrm{O}$ contacts. Clearly, in view of the above observations, no simple relation may be expected between $\Delta E, I$ and $\Delta k$ since the geometrical features which favour large $\Delta E$ or $I$ or $\Delta k$ are rather different. In order to allow a comparison between the properties of the experimental $\mathrm{H}$ bonds and the model $\mathrm{H}$ bonds we have therefore plotted the collected results of $\Delta E, I$ and $\Delta k$ against the H-bond distance, $R$, in Figs. 5, 6 and 7, respectively. It is seen from Fig. 5 that the $\Delta E$ of most experimental $\mathrm{H}$ bonds is $1-2 \mathrm{kcal} \mathrm{mol}^{-1}$ less attractive than the lowest model one (M2). This arises because the model points have a nearly optimal geometry at each $R$ value. The discrepancy is only $1-2 \mathrm{kcal} \mathrm{mol}^{-1}$, as the energy of a crystal has to be as low as possible [7]. Exceptionally weak $H$ bonds are F5 and T3, while A4 and F4 may be considered as borderline cases. T8 is not H-bonded at all.

The IR intensities, $I$, of the experimental $H$ bonds (Fig. 6) stay within about 0.1 a.u. of those of the M2, M9, M10 curve $\left(\chi=0\right.$ and $\left.\theta=50^{\circ}\right)$ which gives almost maximal $I$ at each $R$ value. Large discrepancies from this curve, such as T8, are rare because, at a given $R, I$ is determined mainly by the non-linearity $\chi$ and this is rarely larger than $20^{\circ}$ [7]. The intensities obtained for F4, F5, A4 and T2 are comparatively low, a point to which we return in a later section. 


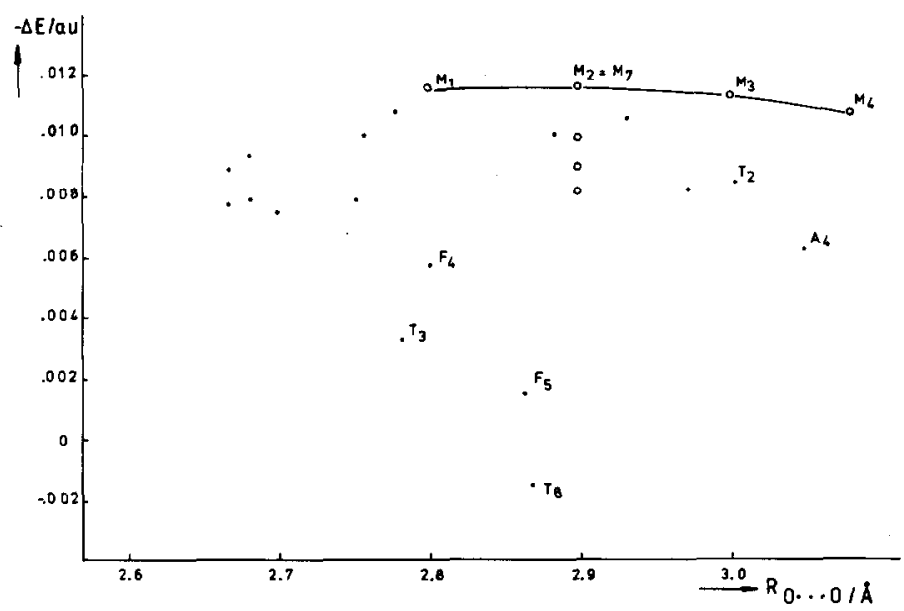

Fig. 5. H-bond energies $\Delta E$ as a function of $R$ for all $\left(\mathrm{H}_{2} \mathrm{O}\right)_{2}$ geometries (O) refers to model geometries $\mathrm{M} 1-\mathrm{M} 10$ ).

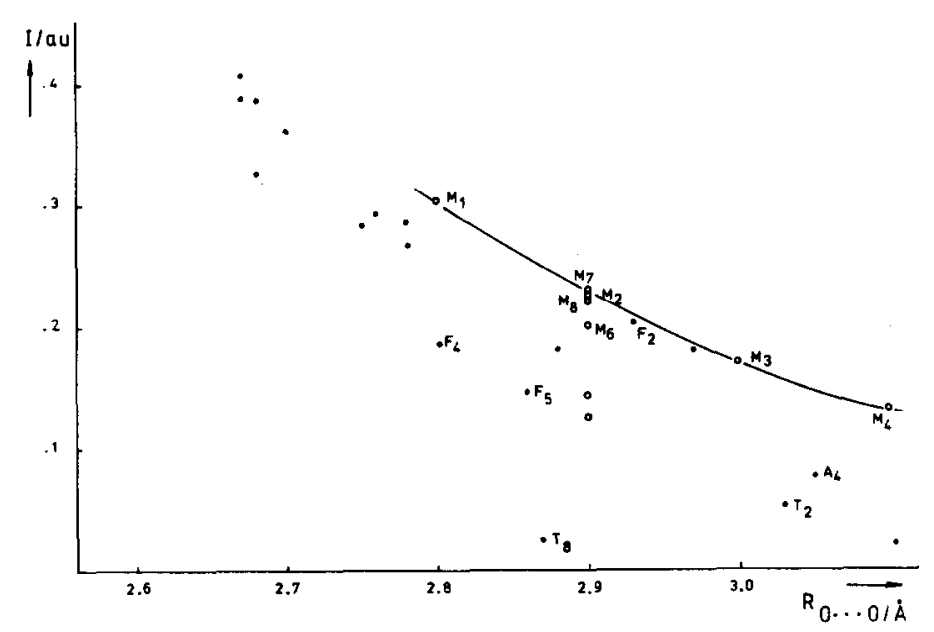

Fig. 6. H-bonded $\mathrm{O}-\mathrm{H}$ IR absorption intensities $I$ as a function of $R$ for all $\left(\mathrm{H}_{2} \mathrm{O}\right)_{2}$ geometries.

It was noted above that for a collection of $\left(\mathrm{H}_{2} \mathrm{O}\right)_{2}$ geometries, $I$ may be proportional to $E^{2}$ and/or $E^{\text {exch }}$. We have obtained plots of these relationships including the model $\mathrm{H}$ bonds, as well as all 17 experimental $\mathrm{H}$ bonds. The best and almost linear correlation is observed between $I$ and $E^{2}$ and this is shown in Fig. 8. Thus, we conclude that the increase in $(\partial \mu / \partial r)$ upon dimerization is primarily due to induction and CT effects (cf. [16]). This conclusion is in keeping with Lindgren and Tegenfeldt's finding that a large increase in $(\partial \mu / \partial r)$ occurs if the $\mathrm{H}_{2} \mathrm{O}$ monomer is placed in an electric field simulating a crystalline environment [17]. 


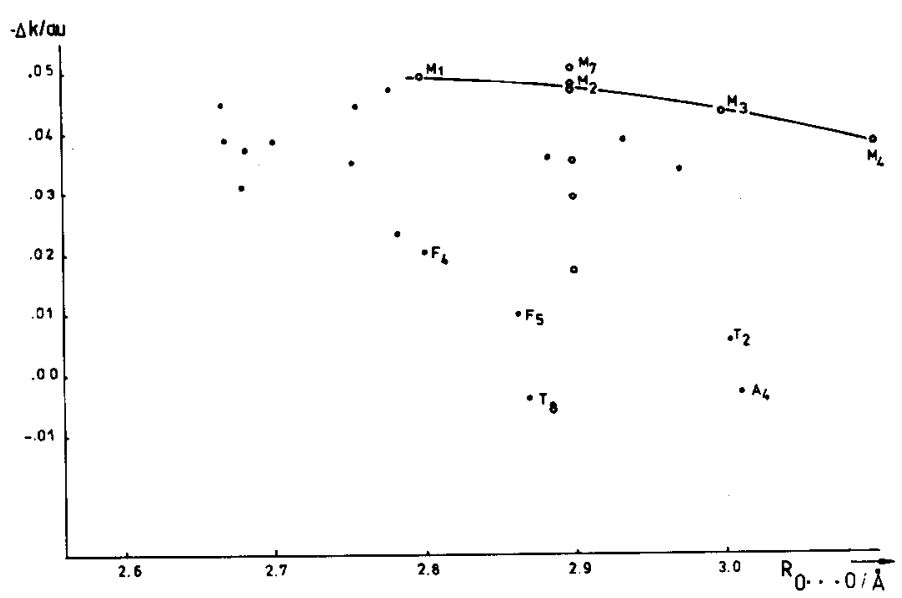

Fig. 7. $\mathrm{H}$-bonded $\mathrm{O}-\mathrm{H}$ force constant change $\Delta k$ as a function of $R$ for all $\left(\mathrm{H}_{2} \mathrm{O}\right)_{2}$ geometries.

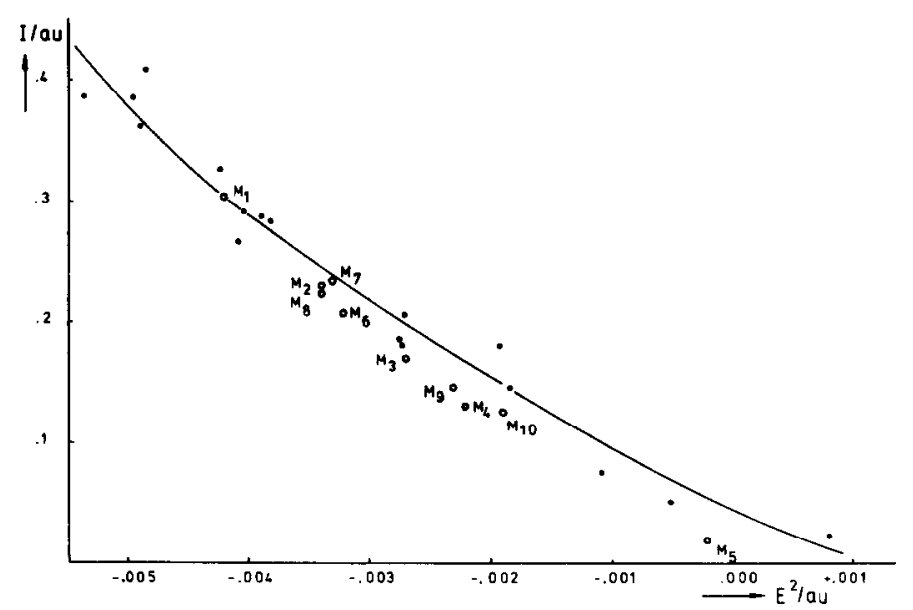

Fig. 8. Correlation between the $\mathrm{OH}$ IR absorption intensities $I$ and the second-order interaction energies $E^{2}$, for all $\left(\mathrm{H}_{2} \mathrm{O}\right)_{2}$ geometries.

Figure 7 shows that the model curve for the geometries M1-M4 gives the maximum $\Delta k$ at a given $R$. At $R=2.6$ or $2.7 \AA$ the experimental points are close to the model curve, in keeping with the observed near-linearity of these bonds. However, for $R \geqslant 2.8 \AA$, many experimental $\mathrm{H}$ bonds (e.g., F5, T2, $\mathrm{T} 8$ and $\mathrm{A} 4$ ) have a $\Delta k$ value which is much smaller than the maximum value at that $R$.

We have verified that for these four contacts the donor $\mathrm{H}$ atom falls on the same side of the $\mathrm{O} \ldots \mathrm{O}$ line as the acceptor bonds, i.e., they are similar 
to $\mathrm{M} 10(\chi=-20)$ which also has a very small $\Delta k$. It is important to emphasize that although these $\mathrm{O}-\mathrm{H} \ldots \mathrm{O}$ contacts have $\Delta k \approx 0$, some of them are strong hydrogen bonds. T2, for example, has a $\Delta E$ value of -0.008 a.u. $\left(5 \mathrm{kcal} \mathrm{mol}^{-1}\right)$. Thus, the Badger-Bauer rule [18], which proposes a linear relation between the enthalpy of a $\mathrm{H}$ bond and the shift in frequency of the $\mathrm{OH}$ bond, does not hold for different orientations of a given $\mathrm{H}$-bonding systems (e.g., $\left.\left(\mathrm{H}_{2} \mathrm{O}\right)_{2}\right)$.

\section{RELATION BETWEEN $\Delta k$ AND $\Delta r$}

In this section we show, by analysing $\Delta k$, how $\Delta k$ may be related to monomer properties and derivatives of the interaction potential. $\Delta k$ may be partitioned into two contributions by writing

$$
\begin{aligned}
\Delta k \equiv k_{\mathrm{d}}-k_{\mathrm{m}} & \equiv\left\{k_{\mathrm{d}}\left(r_{\mathrm{d}}\right)-k_{\mathrm{d}}\left(r_{\mathrm{m}}\right)\right\}+\left\{k_{\mathrm{d}}\left(r_{\mathrm{m}}\right)-k_{\mathrm{m}}\left(r_{\mathrm{m}}\right)\right\} \\
& \equiv \Delta k_{\text {indirect }}+\Delta k_{\text {direct }}
\end{aligned}
$$

These subscripts refer to the fact that the approach of the acceptor has a direct effect upon $k_{\mathrm{d}}$, inasmuch as the second derivative of the total energy in the dimer differs from that in the monomer, even if it is evaluated at the same bond length as in the monomer $\left(r_{\mathrm{m}}\right)$. However, the interaction in the dimer also leads to a change in bond length and, since the monomer energy is an anharmonic function of the bond length, this yields the indirect contribution to $k_{\mathrm{d}}$.

Simple but accurate formulae may be derived which link $\Delta k_{\text {direct }}$ and $\Delta k_{\text {indirect }}$ to the second $\left(\Delta E^{\prime \prime}\right)$ and first $\left(\Delta E^{\prime}\right)$ derivatives of the interaction energy $\Delta E$, respectively. By partitioning the dimer energy according to

$E_{\mathrm{d}}(r) \equiv E_{\mathrm{don}}(r)+E_{\mathrm{acc}}+\Delta E(r)$

and by expanding $E_{\mathrm{don}}$ and $\Delta E$ in a Taylor series around $r_{\mathrm{m}}$, one obtains

$$
\begin{aligned}
E_{\mathrm{d}}(r) & \equiv E_{\mathrm{don}}\left(r_{\mathrm{m}}\right)+\frac{1}{2} k_{\mathrm{m}}\left(r-r_{\mathrm{m}}\right)^{2}+\frac{1}{6} g_{\mathrm{m}}\left(r-r_{\mathrm{m}}\right)^{3}+\ldots \ldots \\
& +E_{\mathrm{acc}}+\Delta E\left(r_{\mathrm{m}}\right)+\Delta E^{\prime}\left(r-r_{\mathrm{m}}\right)+\frac{1}{2} \Delta E^{\prime \prime}\left(r-r_{\mathrm{m}}\right)^{2} \\
& +\frac{1}{6} \Delta E^{\prime \prime \prime}\left(r-r_{\mathrm{m}}\right)^{3}+\ldots \ldots
\end{aligned}
$$

where $\Delta E^{\prime \prime \prime}$ and $g_{\mathrm{m}}$ are the third derivatives in $r$ of $\Delta E$ and $E_{\text {don }}$, respectively. By definition, we have $k_{\mathrm{d}}\left(r_{\mathrm{m}}\right) \equiv E_{\mathrm{d}}^{\prime \prime}\left(r_{\mathrm{m}}\right)$. Therefore, by differentiating eqn. (3) twice, and putting $r=r_{\mathrm{m}}$, we obtain

$\Delta k_{\text {direct }} \equiv k_{\mathrm{d}}\left(r_{\mathrm{m}}\right)-k_{\mathrm{m}}\left(r_{\mathrm{m}}\right)=\Delta E^{\prime \prime}$

i.e., the second derivative of the interaction energy $\Delta E$ gives the direct contribution to $\Delta k$. On the other hand, minus the first derivative, $-\Delta E^{\prime}$, is the force responsible for lengthening or shortening the $\mathrm{OH}$ bond by an amount $\Delta r$. By requiring that in the dimer the first derivative of the dimer energy be zero at $r_{\mathrm{d}}$, one obtains from expression (3), neglecting terms in $\Delta r^{2}$ 
$\Delta r=\frac{-\Delta E^{\prime}}{k_{\mathrm{m}}+\Delta E^{\prime \prime}}+\ldots$

This $\Delta r$ is the cause of $\Delta k_{\text {indirect }}$, for, by differentiating eqn. (3) twice at $r_{\mathrm{d}}$ and again neglecting terms in $\Delta r^{2}$, one finds

$\Delta k_{\text {indirect }}=\Delta r\left(g_{\mathrm{m}}+\Delta E^{\prime \prime \prime}\right)+\ldots$.

That is, since the cubic anharmonicity $\left(g_{m}+\Delta E^{\prime \prime \prime}\right)$ is negative, an increase in bond length leads to a decrease in $k_{\mathrm{d}}$.

These two contributions to $\Delta k$ have been discussed previously by several authors [19], although in the absence of accurate knowledge of the dependence of $\Delta E$ upon the $\mathrm{OH}$ length it has so far not been possible to estimate the relative importance of these contributions. The expressions for $\Delta r$ and $\Delta k_{\text {indirect }}$ may be simplified even further, with some loss in accuracy. For example, by neglecting $\Delta E^{\prime \prime}$ with respect to $k_{\mathrm{m}}$ and $\Delta E^{\prime \prime \prime}$ with respect to $\mathrm{g}_{\mathrm{m}}$, we obtain

$\Delta r \cong\left(-\Delta E^{\prime} / k_{\mathrm{m}}\right)$ and $\Delta k_{\text {indirect }} \cong \Delta r g_{\mathrm{m}}$

which are essentially the same expressions as those of Buckingham [19a]. Of course, the interaction also affects the higher derivatives of $\Delta E$, and thus the anharmonicity of the $\mathrm{OH}$ vibrational motion, as pointed out by Sandorfy [20]. However, an analysis of these effects is beyond the scope of this paper.

Table 1 gives values for $\Delta k_{\text {direct }}$, and the total $\Delta k$ for the 17 observed $\mathrm{O}-\mathrm{H} \cdots \mathrm{O}$ contacts and the ten model geometries. The $\Delta k_{\text {indirect }}$ and $\Delta k_{\text {direct }}$ values were obtained as defined by eqn. (1), by evaluating $k_{\mathrm{d}}$ not only at $r_{\mathrm{d}}$ but also at $r_{\mathrm{m}}$, using four-point interpolation. Inspection of the results shows that, as a rule, the $\Delta k_{\text {direct }}$ values are of the same sign, but smaller than the $\Delta k_{\text {indirect }}$ values, and so $\Delta k$ is usually dominated by $\Delta k_{\text {indirect }}$. The main exceptions occur in cases like T8, A4 and T2 where $\Delta k$ and its components are very small or even positive.

Plots of $\Delta k_{\text {indirect }}$ and the total $\Delta k$ against $\Delta r$ are shown in Fig. 9. For the observed contacts, there is an excellent linear relationship between $\Delta k_{\text {indirect }}$ and $\Delta r$ (Fig. 9(a)). In accordance with eqn. (7) the intercept is zero and the slope (viz. -1.898 a.u.) agrees well with the monomer cubic force constant $\left(g_{m}=-1.866\right.$ up to -1.892 a.u. depending on the donor $\mathrm{HOH}$ angle). This shows that the anharmonicity $\Delta E^{\prime \prime \prime}$ of the interaction energy (cf. eqn. (6)) can be ignored compared to $g_{m}$. The same applies for the $\Delta k_{\text {indirect }}$ versus $\Delta r$ curve for the model $\mathrm{H}$ bonds (not shown), but this curve has a different slope because the four-point grid and hence $g_{m}$ was slightly different.

Unexpectedly, the relation between $\Delta k_{\text {direct }}$ and $\Delta r$ is also roughly linear (although there is a fair amount of scatter) and thus $\Delta k$, being the sum of $\Delta k_{\text {indirect }}$ and $\Delta k_{\text {direct }}$, is roughly linear against $\Delta r($ Fig. 9(b)). This explains why Lindgren and Tegenfeldt found a linear relationship between their calculated values of $\Delta k$ (or in fact $\Delta \nu_{\mathrm{OH}}$ ) and $\Delta r$. 


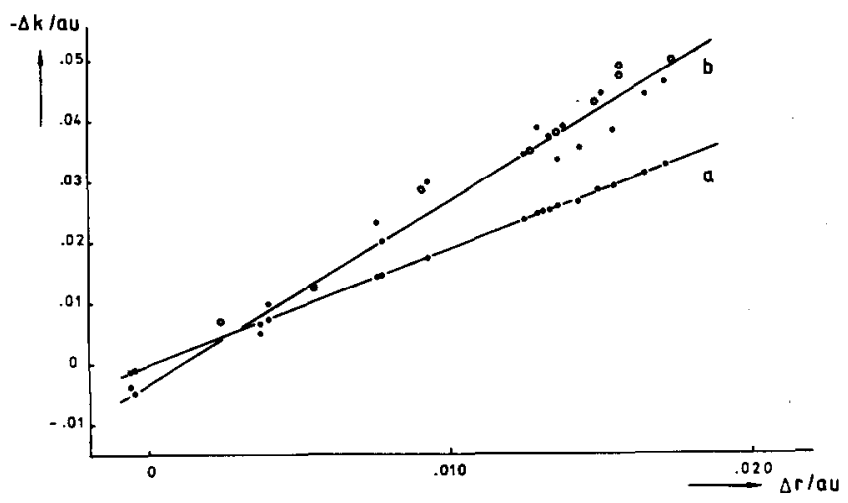

Fig. 9. Linear relations between $\mathrm{H}$-bonded $\mathrm{O}-\mathrm{H}$ lengthening $\Delta r$ and (a) $\Delta k_{\text {indirect }}$; (b) $\Delta k=\Delta k_{\text {indirect }}+\Delta k_{\text {direct }}$.

Having shown that (at least in $\left.\left(\mathrm{H}_{2} \mathrm{O}\right)_{2}\right)$ most of $\Delta k$, and hence most of the observed IR frequency shift, arises from the lengthening of $r_{\mathrm{OH}}$ upon $H$-bond formation, we now analyse via a partitioning of the force $-\Delta E^{\prime}$ which terms in the interaction energy are responsible for this lengthening (cf. eqn. (5)). For this purpose we have evaluated the first derivatives of $E^{\mathrm{Coul}}, E^{\text {exch }}, E^{2}$ and $E^{\text {disp }}$, whose total equals $\Delta E^{\prime}$. The results are tabulated in Table 2. Since in near-linear $\mathrm{H}$ bonds the stretching of $\mathrm{O}-\mathrm{H}$ brings the positive $\mathrm{H}$ atom nearer to the negative acceptor atom, $-\partial E^{\mathrm{Coul}} / \partial r$ is positive, i.e., the classical Coulomb energy tends to lengthen the $\mathrm{OH}$ bond. At long $R$, this is the main contribution to $-\Delta E^{\prime}$ (see, e.g., M5 in Table 2). However, near the dimer equilibrium geometry there is significant exchange repulsion which increases when the $\mathrm{OH}$ length increases [16]. We find that in most cases the influence of the Coulomb energy is more than cancelled by the exchange contribution, resulting in an overall shortening effect of $E^{1}$. The $-\partial E^{\text {dis }} / \partial r$ contribution is seen always to be positive, but very small, ranging only from +0.002 to +0.000 a.u. Stretching the $\mathrm{OH}$ bond makes it more polar and more polarizable which causes an increase of the induction and charge transfer energy. Thus, the effect of $E^{2}$ is also positive (except for T8). In most cases $-\partial E^{2} / \partial r$ is larger than the $-\partial E^{1} / \partial r$ contribution and so $E^{2}$ may be regarded as the principal cause of $\mathrm{O}-\mathrm{H}$ lengthening at short and intermediate $R$ values. The only $\mathrm{O}$... $\mathrm{O}$ contacts which have a small negative $-\Delta E^{\prime}$ are $\mathrm{A} 4$ and T8. For these contacts a slight shortening of the $\mathrm{OH}$ bond is to be expected and, since $\Delta k$ is positive, a shift to higher frequencies. For $T 8$ this arises because each of the $E^{1}, E^{2}$ and $E^{\text {disp }}$ contributions to $-\Delta E^{\prime}$ is small (not unexpectedly, since $\Delta E$ is also very small). By contrast, for A4 the individual contributions are quite large but they cancel. This rationalizes the failure of the Badger-Bauer relation, noted above: if the forces responsible for lengthening $\mathrm{O}-\mathrm{H}$ is small through cancellation of large separate terms the corresponding $\mathrm{O}-\mathrm{H}$... O contact will have a small shift in $\nu_{\mathrm{OH}}$, even though it may be strongly hydrogen bonded. 
TABLE 2

Partitioning of the force, $-\Delta E^{\prime}$, acting on $\mathrm{H}_{\mathrm{d}}$ along $\mathrm{O}-\mathrm{H}_{\mathrm{d}}$ (a.u.)

\begin{tabular}{lllllll}
\hline & $-\left(\partial E^{2} / \partial r\right)$ & $-\left(\partial E^{1} / \partial r\right)$ & $-\left(\partial E^{\text {disp } / \partial r}\right)$ & $-\left(\partial E^{\text {Coul } / \partial r}\right)$ & $-\left(\partial E^{\text {exch }} / \partial r\right)$ & $-\Delta E^{\prime}$ \\
\hline A1 & 0.012 & -0.005 & 0.002 & 0.023 & -0.028 & 0.009 \\
F1 & 0.016 & -0.010 & 0.002 & 0.030 & -0.040 & 0.008 \\
T5 & 0.012 & -0.005 & 0.002 & 0.023 & -0.028 & 0.009 \\
T4 & 0.015 & -0.010 & 0.002 & 0.029 & -0.039 & 0.007 \\
T1 & 0.017 & -0.012 & 0.002 & 0.032 & -0.044 & 0.007 \\
F2 & 0.008 & -0.001 & 0.001 & 0.015 & -0.016 & 0.008 \\
A2 & 0.014 & -0.008 & 0.002 & 0.026 & -0.034 & 0.007 \\
T6 & 0.008 & -0.002 & 0.001 & 0.015 & -0.017 & 0.008 \\
A3 & 0.012 & -0.007 & 0.002 & 0.023 & -0.030 & 0.007 \\
F3 & 0.006 & 0.001 & 0.001 & 0.012 & -0.011 & 0.008 \\
T7 & 0.016 & -0.013 & 0.002 & 0.030 & -0.043 & 0.005 \\
T3 & 0.013 & -0.010 & 0.001 & 0.022 & -0.032 & 0.004 \\
F4 & 0.009 & -0.006 & 0.001 & 0.015 & -0.021 & 0.004 \\
F5 & 0.006 & -0.005 & 0.001 & 0.010 & -0.015 & 0.002 \\
T2 & 0.002 & -0.000 & 0.000 & 0.004 & -0.004 & 0.002 \\
A4 & 0.003 & -0.004 & 0.001 & 0.005 & -0.009 & 0.000 \\
T8 & 0.000 & -0.000 & 0.000 & 0.001 & -0.001 & 0.000 \\
M1 & 0.013 & -0.006 & 0.001 & 0.024 & -0.030 & 0.008 \\
M2 & 0.010 & -0.003 & 0.001 & 0.018 & -0.021 & 0.008 \\
M3 & 0.008 & -0.001 & 0.001 & 0.014 & -0.015 & 0.008 \\
M4 & 0.006 & 0.001 & 0.001 & 0.010 & -0.009 & 0.008 \\
M5 & 0.000 & 0.002 & 0.000 & 0.002 & -0.000 & 0.002 \\
M6 & 0.010 & -0.006 & 0.001 & 0.008 & -0.014 & 0.005 \\
M7 & 0.009 & -0.002 & 0.001 & 0.018 & -0.020 & 0.009 \\
M8 & 0.010 & -0.003 & 0.001 & 0.018 & -0.021 & 0.009 \\
M9 & 0.007 & -0.001 & 0.001 & 0.013 & -0.014 & 0.007 \\
M10 & 0.006 & -0.005 & 0.001 & 0.010 & -0.015 & 0.002 \\
\hline & & & & & &
\end{tabular}

\section{TENTATIVE ASSIGNMENT OF THE OBSERVED HIGH-FREQUENCY PEAKS}

We now turn to the IR spectra observed for the saccharide crystals studied here. We focus attention on the narrow high-frequency peaks $\left(\nu_{\mathrm{OH}} \approx 3600 \mathrm{~cm}^{-1}\right)$ observed in these spectra, since an assignment to free hydroxyl groups [2] proved difficult in some cases [5]. An assignment on the basis of precise frequencies must be ruled out since the $\left(\mathrm{H}_{2} \mathrm{O}\right)_{2}$ model used in our calculations does not allow for non-nearest neighbour interactions. That is, nearly all $\mathrm{O}-\mathrm{H}$... O contacts that we consider form part of a H-bonded network, in which the $\mathrm{O}$ atom in the donor $\mathrm{OH}$ group acts as an acceptor for another neighbouring $\mathrm{OH}$ group and vice versa. Such $\mathrm{H}$-bonded chains are known to absorb at lower frequencies than isolated dimers [21]. Secondly, there may be sources for frequency shifts within the donor molecule itself. Unperturbed $\mathrm{OH}$ frequencies in various monomeric alcohols are known to vary from 3600 to $3650 \mathrm{~cm}^{-1}$ [22]. For saccharides this range is not known but may be expected to be at least as large as for the monomeric alcohols. In our 
calculations we partially account for the differences between the various $\mathrm{OH}$ groups by using the observed $\mathrm{ROH}$ donor angles. These vary from $103^{\circ}$ to $113^{\circ}$, corresponding to a $k$-range of $0.54-0.56$ a.u., i.e., there is a predicted spread in monomer $\mathrm{OH}$ frequencies of about $70 \mathrm{~cm}^{-1}$. However, the experimental angles are not accurately known and the $\mathrm{OH}$ frequencies will also depend on other factors (e.g., small differences in acidity) and so the uncertainty in the calculated monomer frequencies is rather large (perhaps $50 \mathrm{~cm}^{-1}$ ).

Although it is not possible to predict precise frequencies for the narrow bands near $3600 \mathrm{~cm}^{-1}$ their narrowness (less than $20 \mathrm{~cm}^{-1}$ ) can assist in the assignment. Normal H-bonded $\nu_{\mathrm{OH}}$ bands in dense media are broad, primarily through anharmonic coupling and Davydov splitting [23]. For a band to be narrow (at room temperature) the force constants which are responsible for anharmonic coupling of $\nu_{\mathrm{OH}}$ to low-frequency intermolecular modes (e.g., $V_{n n N}$, as discussed in [24]) must be small. This will be the case if $\Delta k$ is small. Moreover, for a band to be narrow, the correlation field splitting by interaction with nearby identical $\mathrm{OH}$ groups at a distance $R$ must also be small. This Davydov splitting is proportional to $I / R^{3}$ and so will be reduced if $I$ is small and $R$ is large. For crystals of primary alcohols, with $R \cong 3 \AA$, splittings of up to $90 \mathrm{~cm}^{-1}$ have been reported [25]. These alcohols are strongly hydrogen bonded, thus their $I$ may be expected to be of the order $I \approx 0.4$ a.u. (like F1, T1, etc.). Table 1 shows that in our calculations the $\mathrm{O}-\mathrm{H} \cdots \mathrm{O}$ contacts with small $\Delta k(<0.02)$ have $I$-values less than about 0.2 a.u. For these contacts the smallest distance to identical sites in the crystal proved to be at least $5 \AA$. Thus, the correlation field splitting for the bands due to these contacts will be only $90(0.2 / 0.4)\left(3^{3} / 5^{3}\right)=9.0 \mathrm{~cm}^{-1}$. We conclude that the narrow high-frequency peaks must be due to contacts having a small $\Delta k$ as well as a small $I$.

The IR spectrum of turanose shows two sharp absorption maxima at frequencies near that of an unperturbed $\mathrm{OH}$ group stretch vibration, viz. at 3610 and $3550 \mathrm{~cm}^{-1}$. Kanters et al. [4] concluded that these peaks correspond to $\mathrm{T} 8$ and $\mathrm{T} 2$, respectively, because $\mathrm{T} 8$ does not act as a donor and T2 has an $\mathrm{O} \cdots \mathrm{O}$ distance of $3.031 \AA$ and an $\mathrm{O}-\mathrm{H} \cdots \mathrm{O}$ angle of $134^{\circ}$. Figures 5, 6 and 7 show that T8 and T2 are indeed the only turanose contacts that may be considered to correspond to these peaks. T8 is not hydrogen bonded and not shifted (having a very small positive $\Delta k$ and $\Delta E$ and a negligible $I$ ) but T2 represents a $\mathrm{H}$ bond ( $\Delta E$ being -0.0084 a.u.) which, however, causes only a very small shift in the $\mathrm{OH}$ frequency $(\Delta k$ being only -0.0050 ) with a small $I$.

The IR spectrum of arabinose reveals a "free" hydroxyl group at $3520 \mathrm{~cm}^{-1}$. Of the four $\mathrm{OH}$...O contacts in arabinose, $\mathrm{A} 4$ is seen to have the smallest but still reasonable $\Delta E$ and the smallest $I$ and it is the only one with a positive $\Delta k$. So, on the basis of our results, we assign the observed peak to A4.

The IR spectrum of $\beta$-D-fructose contains a rather sharp absorption peak 
at $3525 \mathrm{~cm}^{-1}$, well separated from the broad absorption band centered at about $3400 \mathrm{~cm}^{-1}$. Kanters et al. [5] were not able to assign this peak to a particular $\mathrm{O}-\mathrm{H} \cdots \mathrm{O}$ contact by considering the intermolecular $\mathrm{O} \ldots \mathrm{O}$ distances and corresponding $\mathrm{H}$-bonded geometries. From Table 1 it is seen that F5 has the smallest $\Delta E$ (being only -0.0015 a.u.) and also the smallest $I$ and $\Delta k$, so that it seems likely that F5 is responsible for the observed peak.

\section{CONCLUSIONS}

Our conclusions may be summarized as follows:

(1) In $\left(\mathrm{H}_{2} \mathrm{O}\right)_{2}$ most of the observed IR frequency shift arises from the lengthening $\Delta r$ of the donor $\mathrm{OH}$ distance upon $\mathrm{H}$-bond formation. As a result, $\Delta \nu_{\mathrm{OH}}$ is roughly linear in $\Delta r$.

(2) If the force responsible for lengthening $\mathrm{OH}$ is small through cancellation of large separate terms the corresponding $\mathrm{O}-\mathrm{H} . . \mathrm{O}$ contact will have a small shift in $\nu_{\mathrm{OH}}$ even though it may be strongly hydrogen bonded.

(3) Both the change in IR absorption intensity $I$ and the second-order interaction energy $E^{2}$ become larger upon shortening $R_{0} \ldots o$. An almost linear correlation is observed between $I$ and $E^{2}$.

(4) For $\left(\mathrm{H}_{2} \mathrm{O}\right)_{2}$ in a near-equilibrium geometry the IR frequency shift is not very sensitive to $R_{0} \ldots o$, but is very sensitive to departures from H-bond linearity.

(5) The narrow peaks observed near $3600 \mathrm{~cm}^{-1}$ in the spectra of certain hydroxyl-group containing crystals are assigned to $\mathrm{O}-\mathrm{H} \cdots \mathrm{O}$ contacts with small $\Delta \nu$ and low $I$. Some of these are fairly strong hydrogen bonds.

\section{ACKNOWLEDGEMENTS}

We thank Prof. Dr J. H. van der Maas for useful discussions. One of us (D. R. Williams) thanks the Netherlands Organization for Pure Research (ZWO) for the award of a visitors' fellowship (1976-1977).

\section{REFERENCES}

1 G. E. Walrafen, J. Chem. Phys., 44 (1967) 1546; 48 (1968) 244.

2 J. A. Kanters, J. Kroon, A. F. Peerdeman and J. A. Vliegenthart, Nature (London), $222(1969) 370$.

3 J. Lindgren and J. Tegenfeldt, J. Mol. Struct., 20 (1974) 335.

4 J. A. Kanters, W. P. J. Gaykema and G. Roelofsen, Acta Crystallogr., Sect. B, 34 (1978) 1873.

5 J. A. Kanters, G. Roelofsen, B. P. Alblas and I. Meinders, Acta Crystallogr., Sect. B, 33 (1977) 665 . The $\mathrm{H}$ bond geometries were taken from an intermediate stage of refinement.

6 Unpublished X-ray determination. For comparison, see a neutron diffraction study by S. Takagi and G. A. Jeffrey, Acta Crystallogr., Sect. B, 33 (1977) 3033.

7 J. Kroon and J. A. Kanters, J. G. C. M. van Duijneveldt-van de Rijdt, F. B. van Duijneveldt and J. A. Vliegenthart, J. Mol. Struct., 24 (1975) 109. 
8 P. H. Smit, J. L. Derissen and F. B. van Duijneveldt, Mol. Phys., 37 (1979) 501.

9 J. G. C. M. van Duijneveldt-van de Rijdt and F. B. van Duijneveldt, J. Mol. Struct., 35 (1976) 263.

10 IBMOLH, Internal Report, Theoretical Chemistry Group, Utrecht University, 1975.

11 Th. P. Groen, INTACT, Internal Report, Utrecht University, 1975.

12 J. G. C. M. van Duijneveldt-van de Rijdt and F. B. van Duijneveldt, J. Mol. Struct., 89 (1982) 185.

13 B. A. Zilles and W. B. Person, J. Chem. Phys., 79 (1983) 65.

14 K. Nakamoto, J. Margoshes and R. E. Rundle, J. Am. Chem. Soc., 77 (1955) 6480.

15 T. T. Wall and D. F. Hornig, J. Chem. Phys., 43 (1965) 2079.

16 M. Dreyfus and A. Pullman, Theor. Chim. Acta, 19 (1970) 20.

17 J. Almlöf, J. Lindgren and J. Tegenfeldt, J. Mol. Struct., 14 (1972) 427.

18 R. H. Badger and S. H. Bauer, J. Chem. Phys., 5 (1939) 839.

19a A. D. Buckingham, Proc. R. Soc., Ser. A, 248 (1958) 169.

$19 \mathrm{~b}$ F. Shala and J. E. Cahill, J. Chem. Phys., 65 (1976) 5250.

19c S. J. la Placa, W. C. Hamilton, B. Kamb and A. Prakash, J. Chem. Phys., 58 (1973) 567.

20 C. Sandorfy, in P. Schuster, G. Zundel and C. Sandorfy, (Eds.), The Hydrogen Bond, Vol. II, North-Holland, Amsterdam, 1976, p. $613 \mathrm{ff}$.

21 M. C. R. Symons, Chem. Soc. Rev., 12 (1983) 1.

$22 \mathrm{~J}$. H. van der Maas, private communication.

23 A. S. Davydov, Theory of Molecular Excitons, McGraw-Hill, New York, NY, 1962.

24 S. Bratos, J. Lascombe and A. Novak, in H. Ratajczak and W. J. Orville-Thomas (Eds.), Molecular Interactions, Vol. 1, John Wiley, New York, 1980.

25 R. J. Jakobsen, J. W. Brasch, Y. Mikawa, J. Mol. Struct., 1 (1968) 309; I. D. Mikhailov, V. A. Savel'ev, N. D. Sokolov and N. G. Bokii, Phys. Status Solidi, Sect. B, 57 (1973) 719. 